Check for updates

Cite this: Phys. Chem. Chem. Phys., 2019, 21, 25175

Received 30th July 2019, Accepted 18th October 2019

DOI: $10.1039 / c 9 c p 04237 g$

rsc.li/pccp

\section{Enhancement of the supercapacitive properties of laser deposited graphene-based electrodes through carbon nanotube loading and nitrogen doping $\dagger$}

\author{
Ángel Pérez del Pino, (D) *a Marta Rodríguez López, ${ }^{a}$ Mohamed Ahmed Ramadan, (D) ab \\ Pablo García Lebière, ${ }^{a}$ Constantin Logofatu, ${ }^{c}$ Immaculada Martínez-Rovira, ${ }^{d}$ \\ Ibraheem Yousef (D) and Enikö György (D) ae
}

\begin{abstract}
Several technological routes are being investigated for improving the energy storage capability and power delivery of electrochemical capacitors. In this work, ternary hybrid electrodes composed of conducting graphene/reduced graphene oxide (rGO), which store charge mainly through electric double-layer mechanisms, covered by $\mathrm{NiO}$ nanostructures, for adding pseudocapacitance, were fabricated through a matrix assisted pulsed laser evaporation technique. The incorporation of multiwall carbon nanotubes (MWCNTs) provokes an increase of the porosity and thus, a substantial enhancement of the electrodes' capacitance (from 4 to $20 \mathrm{~F} \mathrm{~cm}^{-3}$ at $10 \mathrm{mV} \mathrm{s}^{-1}$ ). Volumetric capacitances of $34 \mathrm{~F} \mathrm{~cm}^{-3}$ were also obtained with electrodes containing just carbon nanotubes coated with $\mathrm{NiO}$ nanostructures. Moreover, the use of nitrogen containing precursors (ammonia, urea) for laser-induced $\mathrm{N}$-doping of the nanocarbons also provokes a notable increase of the capacitance. Remarkably, $\mathrm{N}$-containing groups in rGO-MWCNTs mainly add electric double layer charge storage, pointing to an increase of electrode porosity, whereas redox reactions contribute with a minor diffusion fraction. It was also observed that the loading of carbon nanotubes leads to an increase of diffusion-controlled charge storage mechanisms versus capacitive ones in rGO-based electrodes, the opposite effect being observed in graphene electrodes.
\end{abstract}

\section{Introduction}

The urgent need of exploitation of renewable energy sources, the electrification of transportation and industry sectors, and the expansion of portable electronics require the development of new technologies for energy storage. Great research and innovation efforts are being carried out for the advance in electrochemical energy storage devices, mainly as rechargeable batteries and electrochemical capacitors (also known as supercapacitors, SCs). ${ }^{1,2}$ Electrochemical energy storage systems have specific performances that make them suitable for different types of applications. Thus, while batteries exhibit high

\footnotetext{
${ }^{a}$ Instituto de Ciencia de Materiales de Barcelona, Consejo Superior de Investigaciones Cientificas (ICMAB-CSIC), Campus UAB, 08193 Bellaterra, Spain. E-mail: aperez@icmab.es; Tel: +34935801853

${ }^{b}$ Faculty of Engineering, Helwan University, Helwan, Egypt

${ }^{c}$ National Institute for Materials Physics, PO Box MG 7, 77125 Bucharest, Romania

${ }^{d}$ ALBA Synchrotron, Carrer de la Llum, 2-26, 08290 Cerdanyola del Vallès, Spain

${ }^{e}$ National Institute for Lasers, Plasma and Radiation Physics, PO Box MG 36,

77125 Bucharest, Romania

$\dagger$ Electronic supplementary information (ESI) available. See DOI: 10.1039/c9cp04237g
}

capability for energy storage but at relatively low power, SCs disclose lower energy density but the ability to provide much higher power besides longer cycling stability. The continuous enhancement of SC performance, increasing the energy storage density without substantial detriment of power delivery and lifetime, is allowing a rapid expansion of SCs in diverse sectors, such as consumer electronics, wind energy, electricity grid management, electric vehicles, and high power medical and industrial systems. ${ }^{3,4}$

Generally, the electric energy in SCs can be stored in two ways: (i) by means of electrostatic adsorption of electrolyte ions on the electrode's surface (electric double layer, EDL), and (ii) through surface faradaic oxidation and reduction of electrochemically active entities (pseudocapacitance). Electrostatic mechanisms (EDL) are appropriate for delivering moderate energy at high power. However, surface faradaic reactions (which add pseudocapacitance) exhibit the storage of much higher energy density but at lower power. One strategy with great potential for improving the SC performance is the fabrication of hybrid electrode materials, in which both types of energy storage mechanisms are combined. This kind of electrode is commonly 
formed by a porous composite of nanostructured carbon (EDL) coated with pseudocapacitive materials as electroactive polymers or transition metal oxides. ${ }^{4-8}$ In these materials, nanostructured carbon acts as a conductive scaffold for pseudocapacitive nanomaterials, being able to transport electrons between the pseudocapacitive material and the current collector, and supply EDL capacitance. The doping of carbon materials with heteroatoms such as oxygen, nitrogen, sulphur or boron is also used for adding pseudocapacitance by means of redox reactions and by enhancement in electrical conductivity. ${ }^{9-13}$ On the other hand, the morphology (distribution of pores) of the material is a key factor for its energy storage performance since it determines the ability of electrolyte ions to reach active sites on the electrode's surface contributing to capacitance. ${ }^{14-17}$ Indeed, there are also works that challenge the "conventional view" of pseudocapacitance mechanisms behind the enhancement of capacitance in an N-doped graphitic structure, which point to the increase of EDL contribution due to the formation of ultramicropores. ${ }^{18}$ Therefore, the use of one-dimensional carbon nanotubes (CNTs) to physically separate two-dimensional graphene sheets, which tend to aggregate, appears as a remarkable method for obtaining high surface area nanocarbon electrodes. ${ }^{19-22}$ Further improvement of the capacitance of the graphene-CNT composites can be obtained through doping with nitrogen-containing chemical groups or by decorating them with pseudocapacitive materials (ternary hybrids). ${ }^{23-26}$

Different approaches are also being investigated for the synthesis of new nanostructured materials with improved performance. ${ }^{8,27,28}$ In this respect, laser-based techniques are revealing high potential for the rapid and versatile fabrication of SC electrodes and devices. ${ }^{29-31}$ Besides triggering of photochemical processes, laser methods are based on the fast heating of the irradiated materials, even up to thousands of degrees, leading to extreme, out of thermodynamic equilibrium, chemical reactions and phase changes. Taking advantage of these exceptional properties, a deposition method based on the matrix assisted pulsed laser evaporation (MAPLE) technique was developed for the fabrication of hybrid composite films formed by reduced graphene oxide ( $\mathrm{rGO}$ ) coated with transition metal oxide nanostructures. ${ }^{32-35}$ The complex laser-matter interactions occurring during deposition lead to the synthesis of a rich variety of compounds with different functional properties, even by slight modifications of the laser parameters, the target composition or the chemical environment. ${ }^{36,37}$ In particular, the reactive inverse MAPLE (RIMAPLE) method was able to accomplish the simultaneous chemical conversion and deposition of the irradiated nanomaterials, allowing the fabrication of functional NiO-coated nitrogen-doped rGO-based SC electrodes. ${ }^{38,39}$ In these studies, the composites containing NiO nanostructures exhibited higher stability than those not containing NiO layers, though lower volumetric capacitance probably due to differences in compactness of the films.

In the present paper, and for the first time, ternary hybrid SC electrodes composed of $\mathrm{rGO} /$ graphene and multiwall carbon nanotubes (MWCNTs) decorated with NiO nanostructures were fabricated by RIMAPLE. The addition of the carbon nanotubes allows the opening of ionic transport paths that lead to a substantial increase of the electrodes' volumetric capacitance. Also, the influence on the functional properties and capacitive/ diffusion-limited nature of the electrodes by doping of the carbon nanostructures with nitrogen and replacing rGO by graphene precursor was investigated. Noteworthily, and differing to the conventional interpretation, the obtained results point out that the increase of capacitance obtained by N-doping of rGO flakes/CNTs would essentially arise from an augment of the porosity rather than by redox reactions.

\section{Experimental}

For the MAPLE targets, aqueous dispersions were obtained by mixing graphene oxide (GO) powder (NanoInnova Technologies) with NiO nanoparticles (NPs, Sigma-Aldrich) and multiwall carbon nanotubes (Sigma-Aldrich) doped with carboxylic groups for enhancing their dispersibility. GO powder is composed of less than 15 layer thick sheets and $c a .1 \mu \mathrm{m}^{2}$ lateral size, $\mathrm{NiO}$ NPs are about $50 \mathrm{~nm}$ in diameter and MWCNTs are about $20 \mathrm{~nm}$ in diameter and up to $1 \mu \mathrm{m}$ in length. Deionized water was used as a solvent. Analogous dispersions were also synthesized by replacing GO by graphene (G) powder (NanoInnova Technologies). The concentration of the GO, G and NiO NP precursors was chosen as $5 \mathrm{wt} \%$, and that of MWCNTs was varied up to $2 \mathrm{wt} \%$ to avoid precipitation and too high viscosity of the dispersions. Further dispersions were obtained by adding nitrogen-containing molecules (ammonia, urea and melamine from Sigma-Aldrich) which have been previously proven to provoke laser-induced chemical reactivity and nitrogen doping. ${ }^{38}$ The concentration of ammonia and urea was set to $2 \mathrm{M}$, whereas the melamine concentration was $0.3 \mathrm{M}$ for avoiding precipitation. Next, the dispersions were stirred and sonicated during $20 \mathrm{~min}$ at room temperature for good homogeneity and low aggregation. The MAPLE targets were prepared by filling $3 \mathrm{~mL}$ aluminium holders with the dispersions. Afterwards, the targets were flash frozen by immersion in liquid $\mathrm{N}_{2}$, placed inside the deposition chamber and preserved frozen during the deposition process by using a liquid $\mathrm{N}_{2}$-cooled holder.

The films' depositions were accomplished by submitting 6000 laser pulses $(266 \mathrm{~nm}$ wavelength, $\sim 5 \mathrm{~ns}$ pulse duration, $10 \mathrm{~Hz}$ repetition rate) with $0.4 \mathrm{~J} \mathrm{~cm}^{-2}$ laser fluence to the frozen targets by means of a Brilliant B Nd:YAG laser system (Quantel). During the irradiation process, the laser beam was kept scanning the target surface with an incident angle of $45^{\circ}$. Flexible and conducting $1 \times 1 \mathrm{~cm}^{2}$ substrates made of (i) $0.5 \mathrm{~mm}$-thick copper and (ii) $0.1 \mathrm{~mm}$-thick polypropylene (PP) films coated with sputtered $\mathrm{Au}(20 \mathrm{~nm}) / \mathrm{Cr}(5 \mathrm{~nm})$ layers, were sited in front of the target at a separation distance of $4 \mathrm{~cm}$. The films' growth was carried out in a $20 \mathrm{~Pa} \mathrm{~N}_{2}$ background gas atmosphere after evacuation to a residual pressure of $0.1 \mathrm{~Pa}$.

The morphology of the obtained layers was characterized by extreme high resolution scanning electron microscopy (XHR SEM) through a Magellan 400L system (FEI). The composites' structure was analysed at the nanoscale by means of high resolution transmission electron microscopy (HRTEM) and 
high angle annular dark field scanning TEM (HAADF-STEM) by means of a Tecnai F20 microscope (FEI). The chemical composition of the layers was also investigated by X-ray photoelectron spectroscopy (XPS) and synchrotron-based transreflection mode Fourier transform infrared microspectroscopy (SR-FTIRM). A SPECS spectrometer based on a Phoibos 150 electron energy analyser operating in a constant energy mode was used for XPS analyses, an aluminium anode ( $\mathrm{Al} \mathrm{K} \alpha 1486.74 \mathrm{eV}$ ) being used as a monochromatic X-ray source. Wide range spectra over $1400 \mathrm{eV}$ binding energies were recorded using a $50 \mathrm{eV}$ analyser pass energy. High resolution spectra were also acquired over $20 \mathrm{eV}$ ranges at $10 \mathrm{eV}$ pass energy with an energy resolution of $0.7 \mathrm{eV}$. All the studies were performed in an ultra-high vacuum $\left(\sim 10^{-7} \mathrm{~Pa}\right)$. SR-FTIRM analyses were carried out at the infrared beamline MIRAS of ALBA Synchrotron using a Hyperion 3000 microscope coupled to a Vertex 70 spectrometer (Bruker, Germany). The microscope, equipped with a liquid $\mathrm{N}_{2}$-cooled 50 microns MCT mercury cadmium telluride detector, used a $36 \times$ Schwarzschild objective (NA $=0.65)$. The spectra were acquired using a single masking aperture size of $10 \times 10 \mu \mathrm{m}^{2}$. A collection of 9 spectra per sample was recorded in the $4000-700 \mathrm{~cm}^{-1}$ mid-infrared range at $4 \mathrm{~cm}^{-1}$ spectral resolution with 1024 co-added scans per spectrum. Baselines were subtracted to the spectra for accurate identification of bands.

The electrochemical properties of the electrodes were investigated by means of cyclic voltammetry (CV) and galvanostatic charge-discharge (GCD) cycling in the voltage range of $[0,0.8] \mathrm{V}$ by means of a Keithley 2450-EC Electrochemistry Lab System. The layers deposited on $\mathrm{Au} / \mathrm{Cr} / \mathrm{PP}$ substrates were used as working electrodes in a three-electrode cell. The analyses were carried out in a $0.5 \mathrm{~cm}^{2}$ sample area using a plate material electrochemical cell (Bio-Logic), an $\mathrm{Ag} / \mathrm{AgCl}$ reference electrode
(3 M NaCl internal solution) and a Pt wire counter electrode. $1 \mathrm{M} \mathrm{Na}_{2} \mathrm{SO}_{4}$ aqueous solution was used as electrolyte. CV studies were performed at different sweep rates in the $10-150 \mathrm{mV} \mathrm{s}^{-1}$ range. Galvanostatic charge-discharge cycles were obtained at different current densities (0.03-6 $\mathrm{mA} \mathrm{cm}^{-2}$ ). Electrochemical impedance spectroscopy (EIS) measurements were also performed through a Hioki IM3590 chemical impedance analyser. Impedance values were acquired in the $1 \mathrm{~Hz}-200 \mathrm{kHz}$ frequency range at open circuit potential with a perturbation amplitude of $5 \mathrm{mV}$. An average of 5 measurements per data point was taken in all the electrochemical analyses for minimizing the experimental error. Regression of EIS data to equivalent circuit models was carried out using EIS analyser software.

\section{Results and discussion}

The UV pulsed laser irradiation of the frozen MAPLE targets leads to the prompt heating of the carbon and NiO nanostructures, leading to the explosive boiling of the surrounding water ice and the deposition of the nanostructures on the facing substrate. ${ }^{38}$ The obtained films, about a few micrometres thick, present homogeneous black colour and remain adherent to the substrate after bending. Hereafter, the samples composed by GO-CNT-NiO will be referred to as ( $w t \% \mathrm{GO} / \mathrm{wt} \% \mathrm{MWCNT} / \mathrm{wt} \% \mathrm{NiO}$ ) for identifying the relative concentrations of the different components used in the corresponding MAPLE target. The morphology of the GO-CNTNiO samples was analysed by means of SEM. Fig. 1a shows the morphology of the electrode fabricated without CNTs, (5/0/5), revealing a rough structure composed of arbitrarily distributed GO sheets and tens of to a hundred nm nanoparticles (NiO). The addition of carbon nanotubes provokes a clear change in the film's
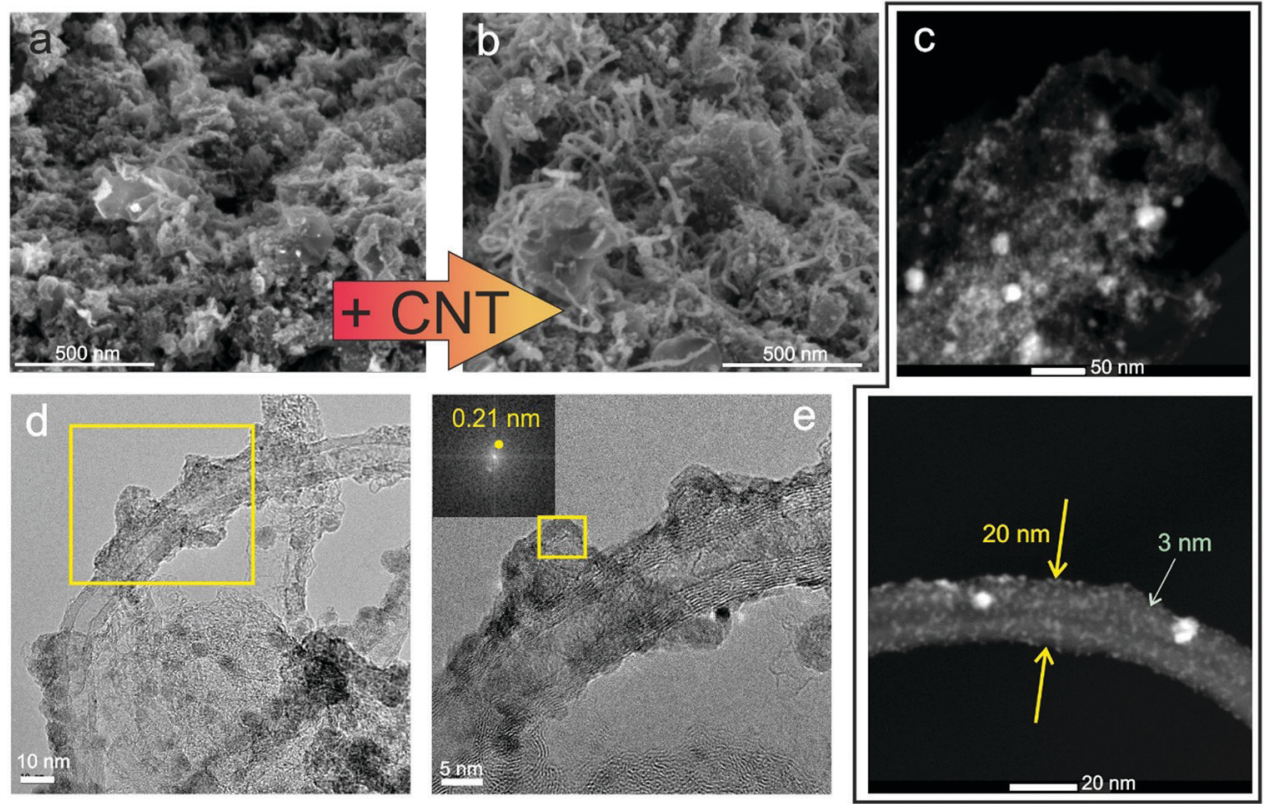

Fig. 1 High-resolution SEM images of (a) GO-CNT-NiO (5/0/5) and (b) (5/1/5). (c) HAADF-STEM images of a GO flake and MWCNT in sample GO-CNT$\mathrm{NiO}$ (5/1/5). (d) High-resolution TEM of sample (5/1/5); (e) zoomed-in area indicated in (d). Inset in (e): FFT of the area indicated by the rectangle. 
morphology even with $1 \mathrm{wt} \%$ of CNTs (Fig. 1b). Randomly oriented CNTs, about $0.5-1 \mu \mathrm{m}$ in length and $c a .20 \mathrm{~nm}$ in diameter, are visible mixed with GO sheets and NiO nanoparticles leading to an increase of the composite's porosity. Higher resolution HAADF-STEM studies reveal the formation of Ni-containing nanostructures (bright regions in Fig. 1c) as small as $1 \mathrm{~nm}$ covering the GO and MWCNT surfaces. The formation of NiO nanostructures with sizes larger and smaller than the initial $\mathrm{NiO}$ NP average diameter $(50 \mathrm{~nm})$ is respectively attributed to coalescence and dewetting mechanisms of molten NiO. High resolution TEM characterization shows that the nanostructures covering both GO flakes and CNTs are crystalline (Fig. 1d and e). Fast Fourier transform analyses of such nanometric regions reveals interplanar distances of $0.21,0.14,0.12$, and $0.10 \mathrm{~nm}$ that are respectively attributed to (200), (220), (222) and (400) crystallographic planes of cubic NiO (Bunsenite; JCPDS 00-0471049). No morphologic differences are observed in samples obtained with ammonia or urea precursors, though the ones obtained with melamine display slightly greater compactness. It is worth noticing that the composites obtained from (0/1/5) MAPLE targets (without GO), show randomly oriented CNTs totally covered by NiO nanostructures to a higher extent than those observed in GO-containing samples (Fig. S1, ESI $\dagger$ ). In this sample, the NiO nanostructures exhibit similar crystalline nature to those of GO-containing samples. However, hundreds of nanometres compact $\mathrm{NiO}$ aggregates are visible in many locations between CNTs (Fig. S1b, ESI†), not visible in GO-containing samples.

Compositional analyses of the GO-CNT-NiO samples were performed by means of XPS and SR-FTIRM. A typical wide scan XPS spectrum is depicted in Fig. 2a, showing C1s, N1s, O1s, and Ni2p and $3 p$ peaks as well as some Auger signals. From these XPS surveys, the overall reduction (at $\% \mathrm{C} / \mathrm{at} \% \mathrm{O}$ ) and nitrogen doping $(100 \times$ at $\% \mathrm{~N} /[\mathrm{at} \% \mathrm{C}+$ at $\% \mathrm{~N}])$ degrees of the nanocarbons were calculated for all the samples (Fig. 2b). As observed, neither an increase of the content of CNTs nor the presence of N-containing precursors in the MAPLE target provoke a significant variation of the reduction degree. Nevertheless, the $\mathrm{N}$-doping degree experiences a noteworthy increase with the respective presence of ammonia, urea and melamine precursors for a given relative composition of GO-CNT-NiO (5/1/5) accounting for a high chemical reactivity of the precursors. Characteristic high resolution XPS spectra of C1s and N1s signals are shown in Fig. 2c. The recorded C1s peaks are deconvoluted to one carboncarbon bond component (284.6 eV, C1), mainly composed of $\mathrm{sp}^{2}$ configuration probably with some contribution of $\mathrm{sp}^{3}$, as well as three carbon-oxygen components centred at 285.7, 286.9 and $288.5 \mathrm{eV}$ respectively attributed to hydroxyl-epoxy (C2), carbonyl (C3) and carboxylic (C4) functionalities. ${ }^{40}$ As observed in Fig. S2a (ESI $\dagger$ ), the integrated area percentage of the C1s components slightly varies between the samples. Though the reduction degree of GO cannot be calculated due to the presence of MWCNTs, previous studies allow us to assume the partial reduction of GO during the deposition of the films. ${ }^{38,41}$ The high resolution N1s signal in the samples obtained from targets without N-containing precursors reveals a very weak and wide peak (Fig. S2b, ESI $\dagger$ ), and is mainly attributed to physisorbed nitrogen from the $\mathrm{N}_{2}$ gas environment during deposition. The signal is much more intense in samples obtained with $\mathrm{N}$-containing precursors (Fig. 2c). In these cases, the N1s signal is deconvoluted into four peaks centred at $c a .398 .1,399.5,401.2$ and $404.5 \mathrm{eV}$ which are respectively associated with the presence of pyridinic $\mathrm{N}-\mathrm{C}$ (N1), pyrrolic $\mathrm{N}-\mathrm{C}$ or amine (N2), graphitic $\mathrm{N}(\mathrm{N} 3)$ and pyridinic $\mathrm{NO}_{x}$ bonds or $\pi$ excitations (N4). ${ }^{36,38}$ Consequently, there must be a contribution of pyridinic and pyrrolic $\mathrm{N}-\mathrm{C}$ bonds to the $\mathrm{C} 2$ and C3 peaks of the C1s spectrum (binding energies at $c a .285 .9$ and $287.3 \mathrm{eV}),{ }^{42,43}$ which may lead to a reduction of the relative quantity of hydroxyl-epoxide and carbonyl groups in these samples. As observed in Fig. S2c (ESI $\dagger$ ), pyrrolic N-C or/and amine (N2) groups are the dominant contribution to N-doping
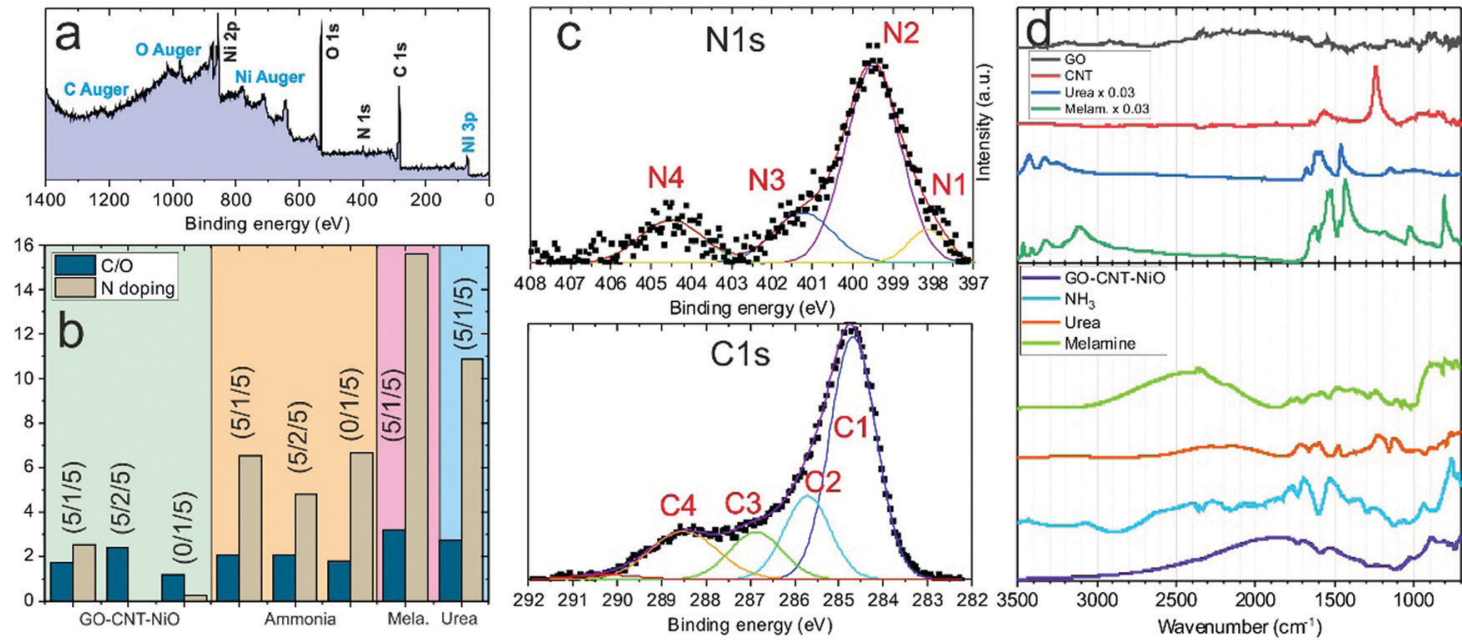

Fig. 2 (a) Wide scan XPS signal of a GO-CNT-NiO (5/1/5)-ammonia sample. (b) Reduction and N-doping degrees of all the samples obtained from XPS surveys. (c) C1s and N1s high-resolution XPS spectra of GO-CNT-NiO (5/1/5)-melamine and (0/1/5)-ammonia samples, respectively. (d) Characteristic FTIRM normalized spectra of raw GO, MWCNT urea and melamine powders as well as deposited samples with (5/1/5) relative concentration. 
in all the samples. Furthermore, the sample (0/1/5)-ammonia, not containing GO, shows a similar $\mathrm{C}-\mathrm{N}$ bond distribution similar to that of samples having GO.

Additional study of the composition of the samples was carried out by means of synchrotron trans-reflection mode-FTIRM. Representative spectra of raw precursors and GO-CNT-NiO (5/1/5) samples are shown in Fig. 2 d. The list of the observed bands and their assignment are presented in Tables S1 and S2 $(\mathrm{ESI} \dagger) .{ }^{44-60}$ As witnessed, raw GO powder reveals very weak characteristic bands probably due to high scattering. The spectrum of CNT material mainly reveals the bands of $\mathrm{CO}$, $\mathrm{COH}$ and $\mathrm{C}-\mathrm{C}$, whereas urea and melamine spectra show the contribution of typical bands of their $\mathrm{N}$-containing groups. The deposited GO-CNT-NiO (5/1/5) films display reflection bands corresponding to different types of $\mathrm{C}-\mathrm{C}, \mathrm{C}-\mathrm{O}$ and $\mathrm{C}-\mathrm{N}$ bonds (Table S2, ESI $\dagger$ ). It is worth noting that a wide-range wavy signal is superimposed on the FTIRM bands due to interferences of IR radiation passing through the studied films. The spectrum of GO-CNT-NiO (5/1/5) mainly shows weak bands corresponding to $\mathrm{C}=\mathrm{C}$, and $\mathrm{C}-\mathrm{O}-\mathrm{C}$, besides the contribution of $\mathrm{C}=\mathrm{O}$ and C-N. Spectra of GO-CNT-NiO (5/1/5) samples obtained with $\mathrm{N}$-containing precursors reveal the presence of additional bands ascribed to several types of carbon-nitrogen chemical groups. Besides, the spectrum of (0/1/5)-ammonia shows the presence of $\mathrm{C}-\mathrm{N}$ bands, similarly to that of GO-CNT-NiO (5/1/5)-ammonia samples with some minor differences, proving the effective nitridation of MWCNTs (Fig. S2d and Table S3, ESI $\dagger$ ). The patterns of the deposited samples do not follow the spectra of the precursor ones, so it is assumed that most of the precursors did react between them and the presence of unreacted molecules can be neglected. These results are in concordance with the significant nitrogen incorporation and bonding type reported by XPS. Neither $\mathrm{C}-\mathrm{H}$ nor $\mathrm{C}-\mathrm{C} \mathrm{sp}^{3}$ bands were detected possibly accounting for the minor formation of structural defects in rGO/CNTs and alkyl chains.

Electrochemical energy storage performance of the electrodes was studied through cyclic voltammetry in the $0.0-0.8 \mathrm{~V}$ voltage and $10-150 \mathrm{mV} \mathrm{s}^{-1}$ sweep rate ranges. Typically, CV plots present a quasi-rectangular shape with no presence of prominent peaks (Fig. 3a). The elevation recorded at the highest potential is triggered by oxygen-evolution reaction in the aqueous electrolyte. The rectangular-like nature of the electrodes' voltammograms, without noticeable redox waves, essentially points toward capacitive (surface) charge storage processes. ${ }^{61,62}$ Therefore, the volumetric capacitance $\left(C_{\mathrm{v}}\right)$ of the electrodes can be calculated from CV data at different sweep rates according to the equation:

$$
C_{\mathrm{v}}=\frac{\oint I(V) \mathrm{d} V}{2 \Delta V s v}
$$

where the integral at the numerator represents the area enclosed in the voltammogram plot (anodic-cathodic carriers), $\Delta V$ is the voltage range $(0.8 \mathrm{~V}), \mathrm{s}$ is the voltage sweep rate and $\mathrm{v}$ is the volume of the active material during the measurements. As observed in Fig. $3 \mathrm{~b}$, the maximum $C_{\mathrm{v}}$ of the (5/0/5) electrode (without CNTs) is about $4 \mathrm{~F} \mathrm{~cm}^{-3}$ (at $10 \mathrm{mV} \mathrm{s}^{-1}$ ). Remarkably, the volumetric capacitance experiences a considerable increase with the incorporation of MWCNTs into the material structure. The maximum values of $C_{\mathrm{v}}$ rise to $\mathrm{ca} .10$ and $20 \mathrm{~F} \mathrm{~cm}^{-3}$ (at $10 \mathrm{mV} \mathrm{s}^{-1}$ ) in samples fabricated with $(5 / 1 / 5)$ and $(5 / 2 / 5)$ relative concentration, respectively. This means a $500 \%$ increase of $C_{\mathrm{v}}$ just by addition of
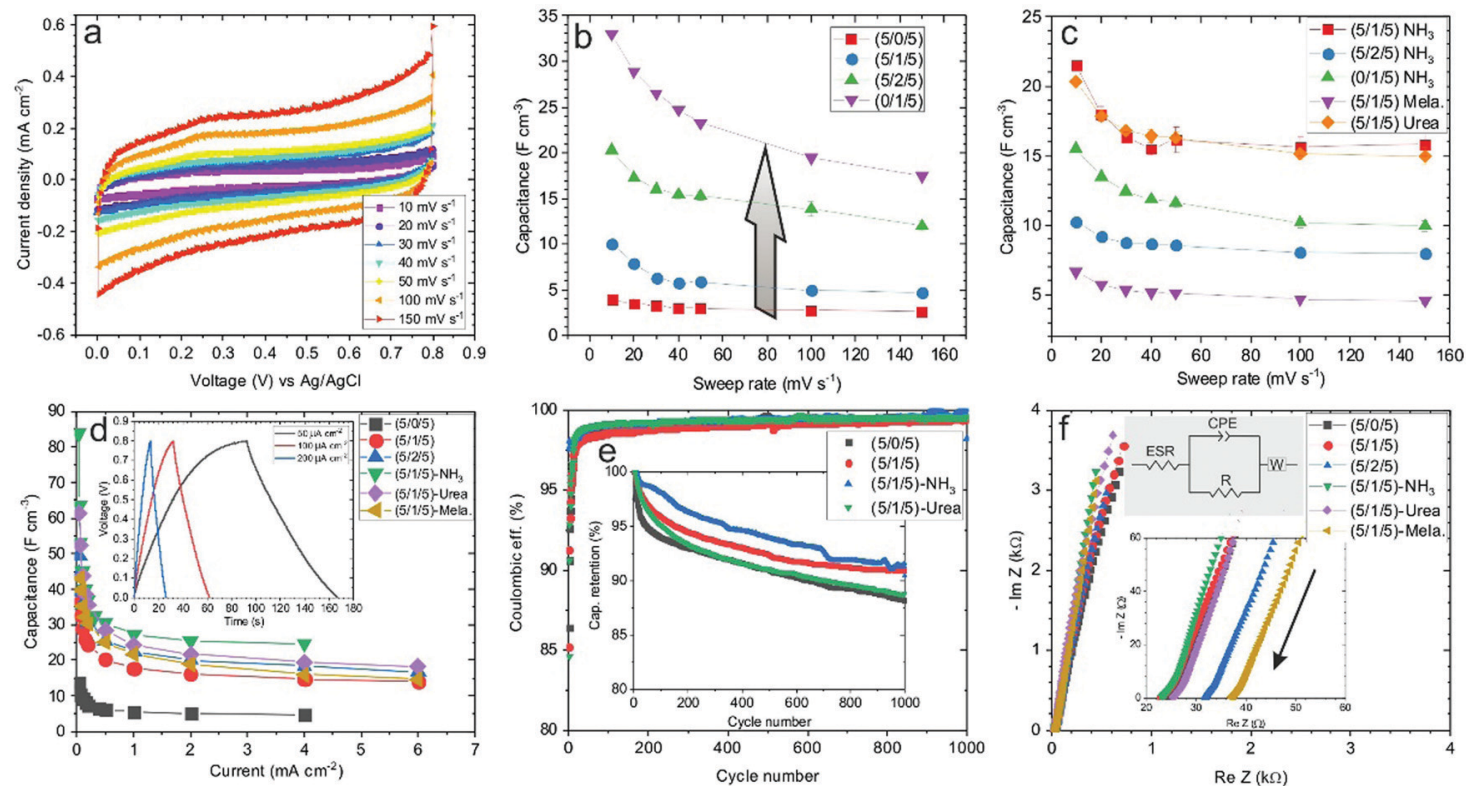

Fig. 3 (a) Cyclic voltammetry plots of the GO-CNT-NiO (5/1/5) sample at different sweep rates. (b) Volumetric capacitance of GO-CNT-NiO samples with different relative concentrations versus sweep rate. (c) Volumetric capacitance of GO-CNT-NiO samples synthesized in the presence of $\mathrm{N}$-containing precursors. (d) Calculated volumetric capacitance versus applied current density of GO-CNT-NiO samples. Inset: Galvanostatic charge-discharge curves of the GO-CNT-NiO (5/1/5) sample at various applied current densities. (e) Coulombic efficiency and capacitance retention (inset) of various GO-CNT-NiO electrodes obtained at ca. $400 \mu \mathrm{A} \mathrm{cm}^{-2}$. (f) Nyquist plots of $\mathrm{GO}-\mathrm{CNT}-\mathrm{NiO}$ samples. Insets: Zoomed-in area at the high frequency region and equivalent circuit used as a model. The arrow indicates the direction of increasing frequency. 
2 wt $\%$ CNTs to the $(5 / 0 / 5)$ target. Furthermore, a capacitance of $34 \mathrm{~F} \mathrm{~cm}^{-3}$ (850\% greater) is even reached in the electrode with no presence of rGO $(0 / 1 / 5)$. The obtained volumetric capacitances could seem quite modest as compared to values of ca. $200-300 \mathrm{~F} \mathrm{~cm}^{-3}$ reported in the literature. ${ }^{21}$ However, taking into account that the mass loading of the obtained films is about $10 \mu \mathrm{g} \mathrm{cm}{ }^{-2}$, the corresponding gravimetric capacitances range from about $100-300 \mathrm{~F} \mathrm{~g}^{-1}$, being very competitive values. ${ }^{19,23,26,63}$ Since the nanostructure and composition of the samples do not significantly differ, we attribute the great change in capacitance to the increase of the film's porosity. A quantitative measurement of the porosity cannot be carried out by the Brunauer-EmmettTeller (BET) method due to the tiny mass loading of the films. However, SEM studies clearly reveal the presence of randomly oriented NiO-coated CNTs inside the films that open channels between rGO sheets improving the electrolyte's ionic access to larger surface area of the material (Fig. 1). 16,17 Consequently, the augmentation of CNT loading leads to an increase of capacitance, up to a certain extent (Fig. 3b). Additionally, the chemical composition takes a complex role in the capacitance of the films (Fig. 3c). GO-CNT-NiO (5/1/5) samples deposited with the presence of ammonia and urea precursors reveal an increased volumetric capacitance of $20-21 \mathrm{~F} \mathrm{~cm}^{-3}$ at $10 \mathrm{mV} \mathrm{s}^{-1}$ (increase of ca. 200\%) even showing different $\mathrm{N}$-doping nature of their structure. Nevertheless, the (5/1/5)-melamine sample, which shows the highest $\mathrm{N}$-doping percentage (essentially pyrrolic $\mathrm{N}$-amine), shows lower capacitance than the (5/1/5) sample. A similar effect was observed previously in rGO-NiO and rGO-NiO-melamine electrodes and was associated with decreasing porosity of the composite material. ${ }^{38}$ Moreover, it has been also reported that the combined action of $\mathrm{O}$ - and $\mathrm{N}$-containing groups such as quinone-, and pyridinic-pyrrolic $\mathrm{N}$ improves the electrochemical energy storage. ${ }^{9}$ Besides, graphitic- $\mathrm{N}$ (not present in melamine sample) has been reported to facilitate the transport of electrons through carbon materials and add strong electron-acceptor nature. ${ }^{10-12}$ All these facts would also contribute to increasing the capacitance of the electrodes obtained with ammonia and urea. Remarkably, an increase of the relative amount of CNTs in the ammonia-based targets, (5/2/5)- and (0/1/5)-ammonia, leads to lower capacitance as compared to their counterparts synthesized without ammonia. However, Fig. 2 and Fig. S2 (ESI $\dagger$ ) reveal similar carbon-oxygen and carbon-nitrogen bonding configuration in these materials. This fact could be caused by: (i) differences in film morphology (porosity), due to different thermal conditions developed at the MAPLE targets during laser irradiation; (ii) different redox behaviour or charge-transfer efficiency of similar $\mathrm{N}$-containing groups depending on their base material, either rGO or MWCNTs; and (iii) subtle differences in the structure of $\mathrm{N}$-containing groups in reduced graphene oxide and carbon nanotubes, not detected by XPS and FTIRM measurements, triggered by differences of reactivity of ammonia with oxygen-containing groups in GO and carboxylic-doped MWCNTs during laser irradiation. ${ }^{64,65}$

Galvanostatic charge-discharge analyses of GO-CNT-NiO samples were performed in the $0.03-6 \mathrm{~mA} \mathrm{~cm}^{-2}$ current density range. The obtained curves display a quasi-linear voltage response with time, leading to the typical triangular-like shape of capacitive materials (Fig. 3d, inset). As expected, an increase of the current density leads to a decrease of the charge/discharge time. The volumetric capacitance of the electrodes was also calculated from GCD data by means of eqn (2):

$$
C_{\mathrm{v}}=\frac{I_{\mathrm{s}} \Delta t_{\mathrm{d}}}{\Delta V d}
$$

being $I_{\mathrm{s}}$ the current density, $\Delta t_{\mathrm{d}}$ the discharge time, $\Delta V$ the voltage range $(0.8 \mathrm{~V})$ and $d$ the thickness of the film. As observed in Fig. 3d, the capacitance drops rapidly with current density at low values of $I_{\mathrm{s}}$, the decay being much slower at currents larger than $0.5 \mathrm{~mA} \mathrm{~cm}{ }^{-2}$. The maximum capacitance, $84 \mathrm{~F} \mathrm{~cm}^{-3}$, is recorded in the (5/1/5)-ammonia electrode at a current density of $30 \mu \mathrm{A} \mathrm{cm} \mathrm{cm}^{-2}$. Besides, the stability of the electrodes was tested applying 1000 consecutive charge-discharge cycles at ca. $0.4 \mathrm{~mA} \mathrm{~cm}^{-2}$. As witnessed in Fig. 3e, the electrodes reveal notable performance since their coulombic efficiency (ratio between charged and discharged carriers) remains at $100 \%$ in the whole cycling range, whereas their capacitive retention slightly decreases with cycling to about $90 \%$ after 1000 cycles. Electrochemical impedance spectroscopy measurements were also carried out for the electrodes. Fig. $3 \mathrm{f}$ presents the Nyquist plots of the samples, which reveal the characteristic straight-line shape at low frequencies with partial semicircle at high frequencies of carbon-based porous electrodes in aqueous electrolytes. ${ }^{39}$ The equivalent circuit depicted in Fig. 3f, based on Randles cell, was used for fitting the following technical parameters: an equivalent series resistance (ESR), which includes the electric resistance of the electrode, electrolyte and circuit contacts; a constant phase element (CPE) related to non-ideal EDL capacitors; a resistor $(R)$ in parallel with the CPE accounting for leakage of charges in the capacitor due to electrochemical reactions (charge transfer); and a Warburg element $(W)$ in series with the capacitor, related to diffusion of ions in the electrolyte. Table S4 (ESI $\dagger$ ) presents the fitted values of these parameters. The ESR of the electrode-electrolyte system ranges from 23-37 $\Omega$, the lowest value belonging to the $(5 / 1 / 5)$ and (5/1/5)-ammonia samples. The n-exponent, related to CPE impedance, indicates the ideality degree of the capacitor. ${ }^{39}$ In all the samples, the $n$ exponent is larger than 0.9 revealing a nearly ideal EDL capacitor performance. The charge transfer (or polarization) resistance $(R)$ is high in all cases $(>50 \mathrm{k} \Omega$ ) accounting for low loss of stored charges, as indicated by the coulombic efficiency measurements in GCD cycling tests. Interestingly, $R$ rises with the loading of MWCNTs, and the (5/1/5)-ammonia sample shows practically no leakages $(R>1 \mathrm{M} \Omega)$. Indeed, the fitted parameters of the (5/1/5)-ammonia electrode point toward a nearly ideal EDL capacitor behaviour. It is also worth noting that the EIS data of all the samples were fitted with a semi-infinite Warburg impedance element, except that of the (5/1/5)-ammonia electrode which had to be fitted using a finite Warburg impedance element for obtaining reliable results.

In the rGO-CNT-NiO hybrid composite that constitutes the electrodes, two types of mechanisms (capacitive and diffusioncontrolled) are expected to contribute to the charge storage. 
Capacitive mechanisms comprehend EDL and pseudocapacitive charge storage on, respectively, graphene domains and electrochemically active sites in (N-doped) rGO flakes and NiO nanostructures. These processes are superficial and fast in nature. On the other hand, diffusion-controlled charge storage encompasses volume mechanisms related to the insertion of species inside the active material. The capacitive and diffusion components of the recorded current during CV measurements are proportional, respectively, to $s$ and $s^{1 / 2} \cdot{ }^{62}$ Therefore, the total current can be expressed as:

$$
i(s, V)=i_{\text {cap }}+i_{\text {dif }}=k_{1} s+k_{2} s^{1 / 2}
$$

Hence, the parameters $k_{1}$ and $k_{2}$ can be calculated by fitting of $i(s, V) / s^{1 / 2}$ versus $s^{1 / 2}$. These parameters allow establishing the amount of capacitive or diffusion current in the voltammograms at any given voltage and sweep rate. Fig. 4 displays calculated capacitive and diffusion components of voltammograms recorded in GO-CNT-NiO (5/0/5), (5/2/5) and (0/1/5) electrodes. As observed, the main contribution in the $(5 / 0 / 5)$ sample comes from the capacitive component in the whole voltage range. The addition of CNTs leads to increased contribution of the diffusion component, as witnessed in the voltammogram of the (5/2/5) sample. Indeed, the evolution of the integrated area ratio of diffusion over capacitive components, shown in Fig. S3 (ESI $\dagger$ ), reveals an increasing trend with the addition of CNTs. As observed, the (0/1/5) sample, which is composed of only NiO-covered CNTs, shows the greatest contribution of the diffusion component over the capacitive one,

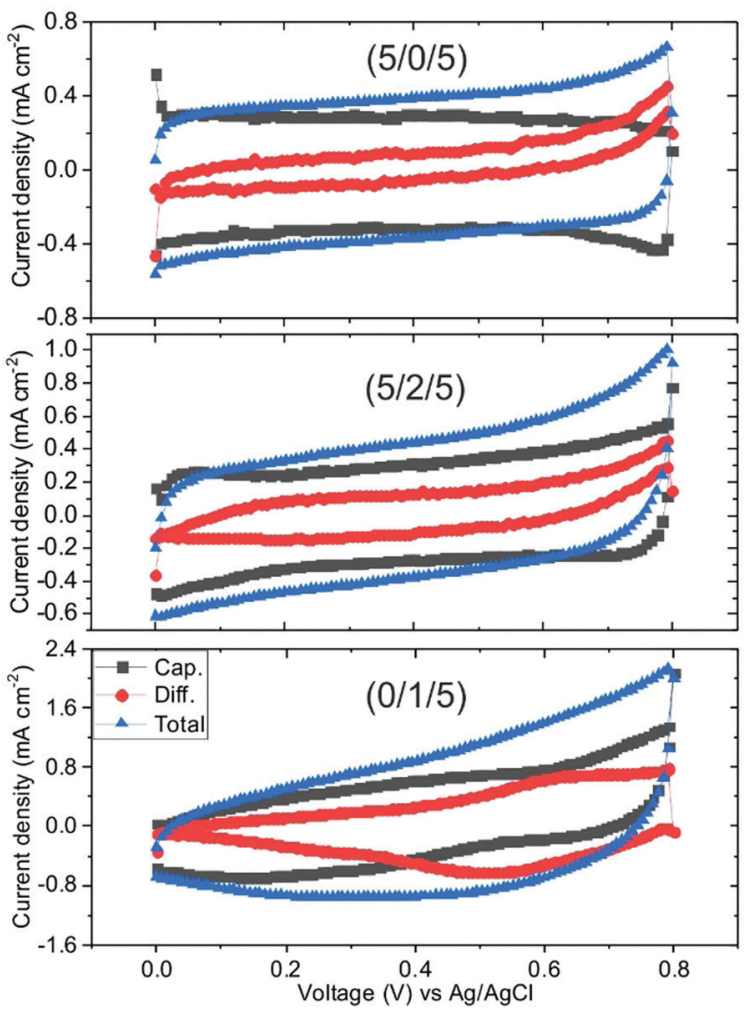

Fig. 4 Decomposition of cyclic voltammetry plots of samples GO-CNT$\mathrm{NiO}(5 / 0 / 5),(5 / 2 / 5)$ and $(0 / 1 / 5)$ taken with $100 \mathrm{mV} \mathrm{s}^{-1}$ into capacitive and diffusion components. especially at larger voltages (Fig. 4 and Fig. S3, ESI $\dagger$ ). The enhanced diffusion nature of NiO-coated CNTs, even enabling larger porosity in the composites than rGO, could be related to thicker NiO-coating on CNTs than on rGO sheets besides the presence of compact NiO aggregates (as inferred from Fig. 1 and Fig. S1, ESI $\dagger$ ). Indeed, the broad and weak waves observed in the diffusion component of the (0/1/5) sample at about $0.5-0.6 \mathrm{~V}$ could be attributed to redox conversions between $\mathrm{NiO}$ and NiOOH species. ${ }^{66,67}$ Therefore, the capacitive or diffusionlimited nature of the material would be somehow driven by the morphology of NiO, which acts as an extrinsic pseudocapacitive material. Besides, typical voltammograms with the calculated capacitive and diffusion-controlled components of $(5 / 1 / 5)$ samples obtained with and without N-containing precursors are displayed in Fig. S4 (ESI $\dagger$ ). As observed, the capacitive component in the composites obtained with $\mathrm{N}$-containing precursors increases (Fig. S3, ESI $\dagger$ ), being more significant in composites obtained with ammonia and urea, which are the ones showing the highest capacitance (Fig. $3 \mathrm{c}$ and d). Moreover, the capacitive component increases practically in the whole voltage range as compared to the (5/1/5) sample. Since pyridinic and pyrrolic $\mathrm{N}$ are associated with edges and nanoholes in the rGO backbone, this augment of the capacitive contribution could be attributed to higher porosity, i.e., accessible surface area, and associated EDL capacitance. ${ }^{18}$ Under comparison, the sample obtained with melamine, which displays slightly greater morphological compactness and the presence of just pyrrolic $\mathrm{N}$-amine groups, reveals somewhat less capacitive component. Furthermore, the diffusion component in N-containing composites is revealed to be slightly larger at low voltages (Fig. S4, ESI $\dagger$ ). This effect has been previously appreciated in electrodes fabricated by RIMAPLE using an imidazole molecule as an N-doping precursor, ${ }^{41}$ and it could be attributed to a diffusion-limited nature of the insertion/extraction processes of active species during faradaic reactions taking place in $\mathrm{N}$-containing functional groups. In summary, $\mathrm{N}$-containing groups add capacitive nature to the electrodes, probably by increasing the active surface area, whereas redox reactions contribute with a minor diffusion-like capacitance mainly at low voltages.

A similar study was accomplished by using graphene (G) sheets instead of GO. Fig. 5a reveals the substantial alteration of the surface morphology of G-CNT-NiO samples with relative concentration of (5/0/5)-without CNTs-, and (5/2/5)-addition of $2 \mathrm{wt} \%$ of CNTs to the target. As observed, the (5/0/5) composite discloses the formation of a compact film with large particles of $\mathrm{NiO}$, up to a few hundred $\mathrm{nm}$ in size, covering the graphene sheets. These large NiO structures, not observed in GO-based materials, would be produced after laser-induced melting, coalescence and recrystallization of aggregated NiO NPs. The aggregation of NiO NPs in the aqueous MAPLE dispersion prior to freezing would be triggered by the hydrophobic nature of the graphene sheets. ${ }^{68}$ Instead, the hydrophilic behaviour of GO flakes would favour their dispersion besides NiO NPs and, thus, the formation of much less compact layers with smaller $\mathrm{NiO}$ structures on rGO sheets after the laser irradiation process. Interestingly, the addition of MWCNTs to the G-NiO NP dispersion 

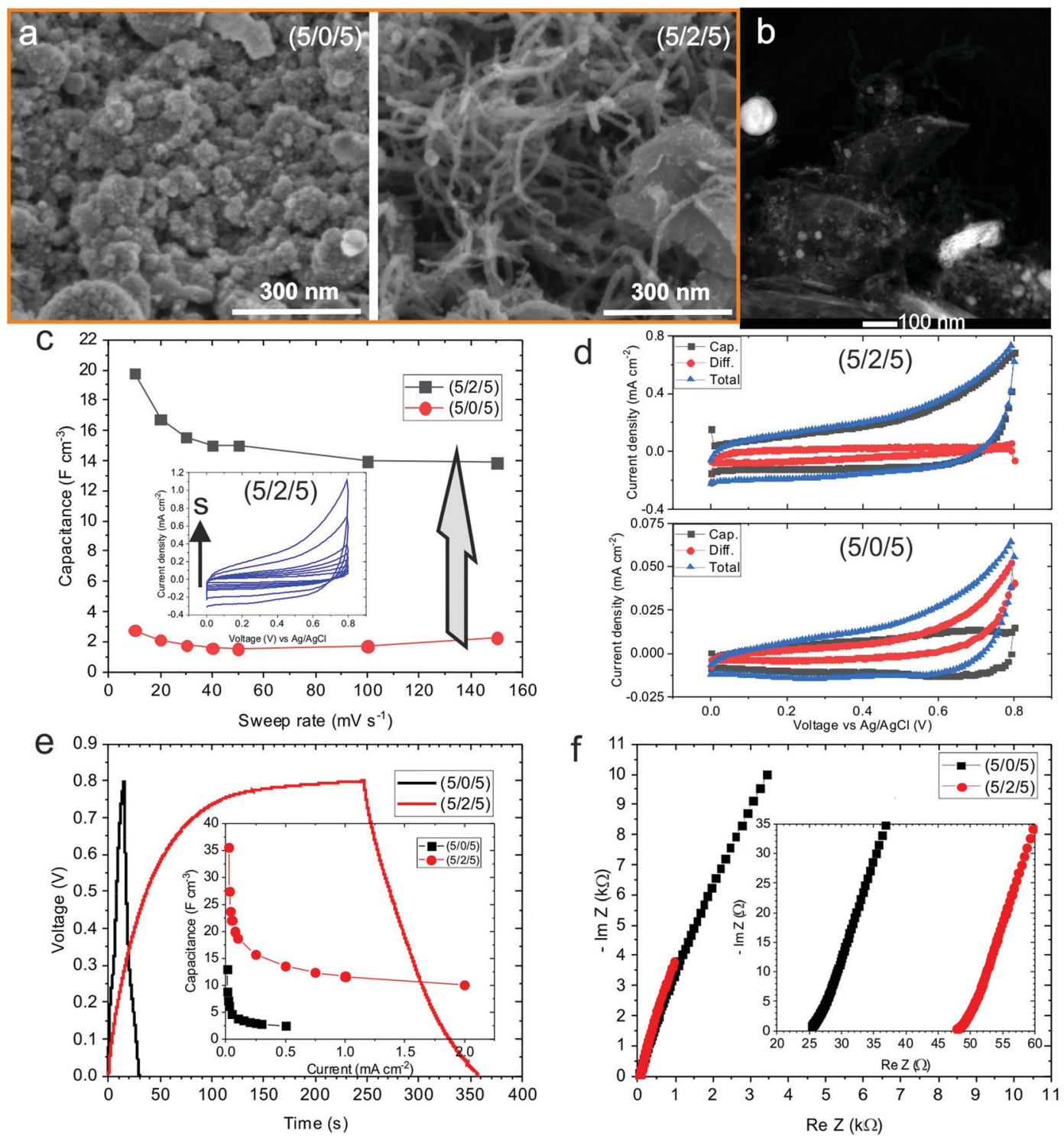

Fig. 5 Study of G-CNT-NiO samples. (a) SEM images of (5/0/5) and (5/2/5). (b) STEM image of (5/2/5). (c) Volumetric capacitance of (5/0/5) and (5/2/5) samples. Inset: Characteristic cyclic voltammetry curves of (5/2/5) at $10-150 \mathrm{mV} \mathrm{s}^{-1}$ sweep rate range. (d) Decomposition of cyclic voltammetry plots of $(5 / 0 / 5)$ and (5/2/5) samples taken with $100 \mathrm{mV} \mathrm{s}^{-1}$ into capacitive and diffusion components. (e) Galvanostatic charge-discharge cycles of (5/0/5) and (5/ 2/5) samples at an applied current of $50 \mu \mathrm{A} \mathrm{cm}{ }^{-2}$. Inset: Calculated volumetric capacitance versus applied current density. (f) Nyquist plots of (5/0/5) and $(5 / 2 / 5)$ samples, being the inset a zoomed-in area of the high frequency data.

leads to the formation of much more porous films composed of NiO-coated graphene sheets and MWCNTs (Fig. 5a). In this case, the average size of the NiO nanostructures is smaller, about tens of nanometres (Fig. 5b). The formation of smaller $\mathrm{NiO}$ nanostructures would be linked to the hydrophilic nature of carboxylic-doped MWCNTs, that would prompt a better dispersibility of the graphene sheets and NiO NPs. Remarkably, after laser deposition, the graphene sheets show a similar structure to that of the G precursor (Fig. S5, ESI $\dagger$ ). No significant formation of laser-induced structural defects is identified. This fact totally differs from the GO material, which experiences a significant structural modification with the appearance of a great quantity of wrinkles and, often, nanoholes after UV laser irradiation. $^{38,41,45}$
Cyclic voltammetry analyses reveal a volumetric capacitance of the G-CNT-NiO (5/0/5) electrode of about $3 \mathrm{~F} \mathrm{~cm}^{-3}$ (at $10 \mathrm{mV} \mathrm{s}^{-1}$ sweep rate) (Fig. 5c). This value is similar to that of the equivalent GO-CNT-NiO (5/0/5) sample (Fig. 3b). As expected, the volumetric capacitance substantially increases after the inclusion of CNTs, reaching $c a .20 \mathrm{~F} \mathrm{~cm}^{-3}$ at $10 \mathrm{mV} \mathrm{s}^{-1}$, about a $660 \%$ increase, which is very similar to that of the equivalent GO-CNT-NiO sample. Therefore, it seems clear that the main limiting factor for the energy storage of these type of layers is the morphology-structure, i.e., the layer porosity. It should be noted that the deposition of G-CNT-NiO material with the presence of N-containing precursors (ammonia, urea, melamine) did not improve the capacitance values due to low reactivity of the precursors with graphene. This fact would indicate that the mechanisms 
leading to the nitrogen incorporation into the graphene structure are indeed promoted by oxygen-containing functionalities of GO. ${ }^{64,65}$ Fig. 5d shows the capacitive and diffusion-limited components of the voltammograms of (5/0/5) and (5/2/5) G-CNT-NiO samples taken with $100 \mathrm{mV} \mathrm{s}^{-1}$. As observed, and to the contrary of the effect observed in the GO-CNT-NiO set of samples, the diffusion-controlled component decreases with the addition of CNTs (Fig. S3, ESI $\dagger$ ). This effect could be explained as the result of less aggregation of $\mathrm{NiO}$ material (shorter diffusion paths) and more contribution of capacitance from graphene surfaces (mainly through double-layer energy storage) with the addition of CNTs. Indeed, by comparison of the capacitive and diffusive components of (5/2/5) GO-CNT-NiO and G-CNT-NiO samples (Fig. 4, 5d and Fig. S3, ESI $\dagger$ ), it is clear that the diffusion contribution of GO-CNT-NiO is larger than that of G-CNT-NiO. Since the size and crystallinity of NiO nanostructures are similar in both samples, this fact would indicate that remaining oxygen functionalities and structural defects present in rGO sheets, but not in G, promote diffusion-limited electrochemical processes.

Similar to GO-based samples, galvanostatic charge-discharge measurements of G-CNT-NiO electrodes show quasi-linear voltage response (Fig. 5e). However, a detailed inspection of the cycles corresponding to the (5/0/5) sample reveals nonlinearities with some levelling at about $0.2 \mathrm{~V}$, which could be an indicator of bulk faradaic reactions (Fig. S6, ESI $\dagger$ ), not observed in the samples containing carbon nanotubes. These faradaic reactions would take place in NiO aggregates as already indicated from studies of capacitive and diffusive components calculated from cyclic voltammetry. As expected, the volumetric capacitance calculated from the charge-discharge data decreases with the applied current density, being about threefold higher in the $(5 / 2 / 5)$ sample than in the $(5 / 0 / 5)$ one. Fig. $5 f$ presents the Nyquist plots of the same samples, revealing slight differences in behaviour. Using the same equivalent circuit as with the GO-CNT-NiO samples, the calculated ESR is about $25 \Omega$ and $48 \Omega$ in the $(5 / 0 / 5)$ and $(5 / 2 / 5)$ electrodes, respectively. Besides, their respective $n$ exponent related to the constant phase element is 0.86 and 0.92 indicating a nearly ideal EDL capacitor behaviour in both cases but greater with the presence of MWCNTs. The value of $\mathrm{R}$ is high in both cases, about $95 \mathrm{k} \Omega$ and $50 \mathrm{k} \Omega$ in the $(5 / 0 / 5)$ and $(5 / 2 / 5)$ electrodes respectively, pointing to low current leakages though being higher with the presence of CNTs. As expected, despite the material aggregation degree, the results point again to EDL electrochemical mechanisms as the main origin of the ternary electrode capacitance.

\section{Conclusions}

The reactive inverse MAPLE fabrication of ternary hybrid electrodes composed of graphene/reduced graphene oxide, multiwall carbon nanotubes and nickel oxide NPs was proven. The relative quantity of MWCNTs allows the tuning of the layer morphology (porosity and $\mathrm{NiO}$ particles dimensions), leading to a boost of the capacitance. Indeed, the electrode morphology seems to be the most important parameter affecting the capacitance. Similar capacitance values are obtained by using either rGO or graphene sheets with equivalent relative material concentrations. Besides, the study of the electrochemical performance of the samples suggests that the augment of capacitance obtained by N-doping of rGO flakes and MWCNTs would mainly come from an increase of the accessible surface area to the active species. Therefore, and contrarily to the most extended conviction, the capacitance of $\mathrm{N}$-doped nanocarbon films would predominantly arise from EDL mechanisms rather than pseudocapacitive ones. The results also demonstrate that the NiO material acts as an extrinsic pseudocapacitive material, adding battery-like behaviour in aggregated form and pseudocapacitive nature when crystallized in a nanometric size.

\section{Conflicts of interest}

There are no conflicts to declare.

\section{Acknowledgements}

The authors acknowledge the financial support of the Spanish Ministry of Economy, Industry and Competitiveness under the project ENE2017-89210-C2-1-R, and support from AGAUR of Generalitat de Catalunya through the project 2017 SGR 1086. ICMAB acknowledges financial support from the Spanish Ministry of Economy and Competitiveness, through the "Severo Ochoa" Programme for Centres of Excellence in R\&D (SEV2015-0496). FTIRM experiments were performed at the MIRAS beamline at the ALBA Synchrotron with the collaboration of ALBA staff. The authors acknowledge support of the publication fee by the CSIC Open Access Publication Support Initiative through its Unit of Information Resources for Research (URICI).

\section{References}

1 X. Zhang, X. Cheng and Q. Zhang, Nanostructured energy materials for electrochemical energy conversion and storage: a review, J. Energy Chem., 2016, 25(6), 967-984.

2 Z. Wang, W. Zhang, X. Li and L. Gao, Recent progress in flexible energy storage materials for lithium-ion batteries and electrochemical capacitors: a review, J. Mater. Res., 2016, 31(12), 1648-1664.

3 F. A. J. R. Miller, Electrochemical Capacitors: Challenges and Opportunities for Real-World Applications, Electrochem. Soc. Int., 2008, 17, 53-57.

4 A. Muzaffar, M. B. Ahamed, K. Deshmukh and J. Thirumalai, A review on recent advances in hybrid supercapacitors: design, fabrication and applications, Renewable Sustainable Energy Rev., 2019, 101, 123-145.

5 D. A. C. Brownson, D. K. Kampouris and C. E. Banks, An overview of graphene in energy production and storage applications, J. Power Sources, 2011, 196(11), 4873-4885.

6 Z.-S. Wu, G. Zhou, L.-C. Yin, W. Ren, F. Li and H.-M. Cheng, Graphene/metal oxide composite electrode materials for energy storage, Nano Energy, 2012, 1(1), 107-131. 
7 L. L. Zhang and X. S. Zhao, Carbon-based materials as supercapacitor electrodes, Chem. Soc. Rev., 2009, 38(9), 2520-2531.

8 G. Wang, L. Zhang and J. Zhang, A review of electrode materials for electrochemical supercapacitors, Chem. Soc. Rev., 2012, 41(2), 797-828.

9 D. Hulicova-Jurcakova, M. Seredych, G. Q. Lu and T. J. Bandosz, Combined Effect of Nitrogen- and OxygenContaining Functional Groups of Microporous Activated Carbon on its Electrochemical Performance in Supercapacitors, Adv. Funct. Mater., 2009, 19(3), 438-447.

10 Y.-H. Lee, K.-H. Chang and C.-C. Hu, Differentiate the pseudocapacitance and double-layer capacitance contributions for nitrogen-doped reduced graphene oxide in acidic and alkaline electrolytes, J. Power Sources, 2013, 227, 300-308.

11 D. Yu, Q. Zhang and L. Dai, Highly Efficient Metal-Free Growth of Nitrogen-Doped Single-Walled Carbon Nanotubes on Plasma-Etched Substrates for Oxygen Reduction, J. Am. Chem. Soc., 2010, 132(43), 15127-15129.

12 S. Yang, L. Zhi, K. Tang, X. Feng, J. Maier and K. Muellen, Efficient Synthesis of Heteroatom ( $\mathrm{N}$ or S)-Doped Graphene Based on Ultrathin Graphene Oxide-Porous Silica Sheets for Oxygen Reduction Reactions, Adv. Funct. Mater., 2012, 22(17), 3634-3640.

13 D.-Y. Yeom, W. Jeon, N. D. K. Tu, S. Y. Yeo, S.-S. Lee, B. J. Sung, H. Chang, J. A. Lim and H. Kim, High-concentration boron doping of graphene nanoplatelets by simple thermal annealing and their supercapacitive properties, Sci. Rep., 2015, 5, 9817.

14 X. Zhao, C. M. Hayner, M. C. Kung and H. H. Kung, Flexible Holey Graphene Paper Electrodes with Enhanced Rate Capability for Energy Storage Applications, ACS Nano, 2011, 5(11), 8739-8749.

15 T. Kim, G. Jung, S. Yoo, K. S. Suh and R. S. Ruoff, Activated Graphene-Based Carbons as Supercapacitor Electrodes with Macro- and Mesopores, ACS Nano, 2013, 7(8), 6899-6905.

16 E. Frackowiak, G. Lota, J. Machnikowski, C. Vix-Guterl and F. Beguin, Optimisation of supercapacitors using carbons with controlled nanotexture and nitrogen content, Electrochim. Acta, 2006, 51(11), 2209-2214.

17 D.-W. Wang, F. Li, M. Liu, G. Q. Lu and H.-M. Cheng, 3D aperiodic hierarchical porous graphitic carbon material for high-rate electrochemical capacitive energy storage, Angew. Chem., Int. Ed., 2008, 47(2), 373-376.

18 X. Wu, J. Zhou, W. Xing, Y. Zhang, P. Bai, B. Xu, S. Zhuo, Q. Xue and Z. Yan, Insight into high areal capacitances of low apparent surface area carbons derived from nitrogenrich polymers, Carbon, 2015, 94, 560-567.

19 D. Yu and L. Dai, Self-Assembled Graphene/Carbon Nanotube Hybrid Films for Supercapacitors, J. Phys. Chem. Lett., 2010, 1(2), 467-470.

20 S. K. Kandasamy and K. Kandasamy, Recent Advances in Electrochemical Performances of Graphene Composite (Graphene-Polyaniline/Polypyrrole/Activated Carbon/Carbon Nanotube) Electrode Materials for Supercapacitor: A Review, J. Inorg. Organomet. Polym. Mater., 2018, 28(3), 559-584.
21 N. Diez, C. Botas, R. Mysyk, E. Goikolea, T. Rojo and D. Carriazo, Highly packed graphene-CNT films as electrodes for aqueous supercapacitors with high volumetric performance, J. Mater. Chem. A, 2018, 6(8), 3667-3673.

22 Q. Cheng, J. Tang, J. Ma, H. Zhang, N. Shinya and L.-C. Qin, Graphene and carbon nanotube composite electrodes for supercapacitors with ultra-high energy density, Phys. Chem. Chem. Phys., 2011, 13(39), 17615-17624.

23 S. N. Faisal, C. M. Subramaniyam, E. Haque, M. M. Islam, N. Noorbehesht, A. K. Roy, M. S. Islam, H. K. Liu, S. X. Dou, A. T. Harris and A. I. Minett, Nanoarchitectured NitrogenDoped Graphene/Carbon Nanotube as High Performance Electrodes for Solid State Supercapacitors, Capacitive Deionization, Li-Ion Battery, and Metal-Free Bifunctional Electrocatalysis, ACS Appl. Energy Mater., 2018, 1(10), 5211-5223.

24 Z. Lei, F. Shi and L. Lu, Incorporation of $\mathrm{MnO}_{2}$-Coated Carbon Nanotubes between Graphene Sheets as Supercapacitor Electrode, ACS Appl. Mater. Interfaces, 2012, 4(2), 1058-1064.

25 W. Li, H. Xu, M. Cui, J. Zhao, F. Liu and T. Liu, Synthesis of sulfonated graphene/carbon nanotubes/manganese dioxide composite with high electrochemical properties, Ionics, 2019, 25(3), 999-1006.

26 R. Zeng, H. Deng, Y. Xiao, J. Huang, K. Yuan and Y. Chen, Cross-linked graphene/carbon nanotube networks with polydopamine "glue" for flexible supercapacitors, Compos. Commun., 2018, 10, 73-80.

27 R. Kumar, R. K. Singh, R. Savu, P. K. Dubey, P. Kumar and S. A. Moshkalev, Microwave-assisted synthesis of voidinduced graphene-wrapped nickel oxide hybrids for supercapacitor applications, RSC Adv., 2016, 6(32), 26612-26620.

28 T. Chen and L. Dai, Flexible supercapacitors based on carbon nanomaterials, J. Mater. Chem. A, 2014, 2(28), 10756-10775.

29 D. Shen, G. Zou, L. Liu, W. Zhao, A. Wu, W. W. Duley and Y. N. Zhou, Scalable High-Performance Ultraminiature Graphene Micro-Supercapacitors by a Hybrid Technique Combining Direct Writing and Controllable Microdroplet Transfer, ACS Appl. Mater. Interfaces, 2018, 10(6), 5404-5412.

30 W. Gao, N. Singh, L. Song, Z. Liu, A. L. M. Reddy, L. Ci, R. Vajtai, Q. Zhang, B. Wei and P. M. Ajayan, Direct laser writing of micro-supercapacitors on hydrated graphite oxide films, Nat. Nanotechnol., 2011, 6(8), 496-500.

31 X.-Y. Fu, Z.-D. Chen, Y.-L. Zhang, D.-D. Han, J.-N. Ma, W. Wang, Z.-R. Zhang, H. Xia and H.-B. Sun, Direct laser writing of flexible planar supercapacitors based on GO and black phosphorus quantum dot nanocomposites, Nanoscale, 2019, 11(18), 9133-9140.

32 R. Ivan, C. Popescu, A. Perez del Pino, I. Yousef, C. Logofatu and E. Gyorgy, Laser-induced synthesis and photocatalytic properties of hybrid organic-inorganic composite layers, J. Mater. Sci., 2019, 54(5), 3927-3941.

33 I. Camps, M. Borlaf, M. T. Colomer, R. Moreno, L. Duta, C. Nita, A. Perez del Pino, C. Logofatu, R. Serna and E. Gyorgy, Structure-property relationships for Eu doped $\mathrm{TiO}_{2}$ thin films grown by a laser assisted technique from colloidal sols, RSC Adv., 2017, 7(60), 37643-37653. 
34 S. M. O’Malley, J. Tomko, A. Perez del Pino, C. Logofatu and E. Gyoergy, Resonant Infrared and Ultraviolet Matrix-Assisted Pulsed Laser Evaporation of Titanium Oxide/Graphene Oxide Composites: A Comparative Study, J. Phys. Chem. C, 2014, 118(48), 27911-27919.

35 E. Gyorgy, C. Logofatu, A. Perez del Pino, A. Datcu, O. Pascu and R. Ivan, Enhanced UV- and visible-light driven photocatalytic performances and recycling properties of graphene oxide/ZnO hybrid layers, Ceram. Int., 2018, 44(2), 1826-1835.

36 A. Queralto, A. Perez del Pino, C. Logofatu, A. Datcu, R. Amade, I. Alshaikh, E. Bertran, I. Urzica and E. Gyorgy, MAPLE synthesis of reduced graphene oxide/silver nanocomposite electrodes: influence of target composition and gas ambience, J. Alloys Compd., 2017, 726, 1003-1013.

37 A. Queralto, A. Perez del Pino, C. Logofatu, A. Datcu, R. Amade, E. Bertran-Serra and E. Gyorgy, Reduced graphene oxide/iron oxide nanohybrid flexible electrodes grown by laser-based technique for energy storage applications, Ceram. Int., 2018, 44(16), 20409-20416.

38 A. Perez del Pino, A. Martinez Villarroya, A. Chuquitarqui, C. Logofatu, D. Tonti and E. Gyorgy, Reactive laser synthesis of nitrogen-doped hybrid graphene-based electrodes for energy storage, J. Mater. Chem. A, 2018, 6(33), 16074-16086.

39 A. P. del Pino, M. A. Ramadan, P. G. Lebiere, R. Ivan, C. Logofatu, I. Yousef and E. Gyorgy, Fabrication of graphene-based electrochemical capacitors through reactive inverse matrix assisted pulsed laser evaporation, Appl. Surf. Sci., 2019, 484, 245-256.

40 A. Perez del Pino, E. Gyoergy, C. Logofatu, J. Puigmarti-Luis and W. Gao, Laser-induced chemical transformation of graphene oxide-iron oxide nanoparticles composites deposited on polymer substrates, Carbon, 2015, 93, 373-383.

41 M. A. R. A. Pérez del Pino, P. Garcia Lebière, R. Ivan, C. Logofatu, I. Yousef and E. György, Fabrication of graphene-based electrochemical capacitors through reactive inverse matrix assisted pulsed laser evaporation, Appl. Surf. Sci., 2019, 484, 245-256.

42 D. Kepic, S. Sandoval, A. Perez del Pino, E. Gyorgy, L. Cabana, B. Ballesteros and G. Tobias, Nanosecond Laser-Assisted Nitrogen Doping of Graphene Oxide Dispersions, ChemPhysChem, 2017, 18(8), 935-941.

43 T. I. T. Okpalugo, P. Papakonstantinou, H. Murphy, J. McLaughlin and N. M. D. Brown, High resolution XPS characterization of chemical functionalised MWCNTs and SWCNTs, Carbon, 2005, 43(1), 153-161.

44 X. Mao, J. Xu, X. He, W. Yang, Y. Yang, L. Xu, Y. Zhao and Y. Zhou, All-solid-state flexible microsupercapacitors based on reduced graphene oxide/multi-walled carbon nanotube composite electrodes, Appl. Surf. Sci., 2018, 435, 1228-1236.

45 A. Perez del Pino, A. Gonzalez-Campo, S. Giraldo, J. Peral, E. Gyorgy, C. Logofatu, A. J. deMello and J. Puigmarti-Luis, Synthesis of graphene-based photocatalysts for water splitting by laser-induced doping with ionic liquids, Carbon, 2018, 130, 48-58.

46 V. S. N. Sharma, Y. Jain, M. Kumari, R. Gupta, S. K. Sharma and K. Sachdev, Synthesis and Characterization of Graphene Oxide (GO) and Reduced Graphene Oxide (rGO) for Gas Sensing Application, Macromol. Symp., 2017, 376, 1700006.
47 R. Trusovas, G. Raciukaitis, G. Niaura, J. Barkauskas, G. Valusis and R. Pauliukaite, Recent Advances in Laser Utilization in the Chemical Modification of Graphene Oxide and Its Applications, Adv. Opt. Mater., 2016, 4(1), 37-65.

48 G. Li, FT-IR studies of zeolite materials: characterization and environmental applications, University of Iowa, 2005.

49 R. A. Nyquist and S. L. Fiedler, Infrared study of 5-membered and 6-membered type cyclic imides, Vib. Spectrosc., 1995, 8(3), 365-386.

50 E. J. Barton, S. N. Yurchenko, J. Tennyson, S. Clausen and A. Fateev, High-resolution absorption measurements of $\mathrm{NH}_{3}$ at high temperatures: 2100-5500 $\mathrm{cm}^{(-1)}$, J. Quant. Spectrosc. Radiat. Transfer, 2017, 189, 60-65.

51 J. Grabska, K. B. Bec, C. G. Kirchler, Y. Ozaki and C. W. Huck, Distinct Difference in Sensitivity of NIR vs. IR Bands of Melamine to Inter-Molecular Interactions with Impact on Analytical Spectroscopy Explained by Anharmonic Quantum Mechanical Study, Molecules, 2019, 24(7), 1402.

52 M. Auguscik, M. Kuranska, A. Prociak, W. Karalus, K. Lipert and J. Ryszkowska, Production and characterization of poly(urea-urethane) elastomers synthetized from rapeseed oil-based polyols Part I. Structure and properties, Polimery, 2016, 61(7-8), 490-498.

53 E. J. V. R. M. Ragamathunnisa, R. Padmavathy and N. Radha, Spectroscopic study on Thiourea and Thiosemicarbazide in Non-aqueous media, IOSR, J. Appl. Phys., 2013, 4, 5-8.

54 Z. P. A. T. Urbanski, The Infra-red Absorption Spectrum and Structure of Urea, Bulletin De L'Academie Polonaise Des Sciences Serle des sciences chimlques, 1962, 3, 113-120.

55 A. Keuleers, H. O. Desseyn, B. Rousseau and C. Van Alsenoy, Vibrational analysis of urea, J. Phys. Chem. A, 1999, 103(24), 4621-4630.

56 L. Zhong and K. Yun, Graphene oxide-modified ZnO particles: synthesis, characterization, and antibacterial properties, Int. J. Nanomed., 2015, 10, 79-92.

57 M. D. Frogley, C. Wang, G. Cinque and A. H. Barber, Polarised infrared microspectroscopy of edge-oriented graphene oxide papers, Vib. Spectrosc., 2014, 75, 178-183.

58 E. D. Grezia, Majorana and the Investigation of Infrared Spectra of Ammonia, Electron. J. Theor. Phys., 2006, 10, 225-238.

59 IR spectrum table - Sigma Aldrich, https:/www.sigmaal drich.com/technical-documents/articles/biology/irspectrum-table.html, 2019.

60 P. H. H. Fischer and C. A. McDowell, The infrared absorption spectra of urea-hydrocarbon adducts, Can. J. Chem., 1960, 38(2), 187-193.

61 Y. Gogotsi and R. M. Penner, Energy Storage in Nanomaterials Capacitive Pseudocapacitive, or Battery-like?, ACS Nano, 2018, 12(3), 2081-2083.

62 J. Liu, J. Wang, C. Xu, H. Jiang, C. Li, L. Zhang, J. Lin and Z. X. Shen, Advanced Energy Storage Devices: Basic Principles, Analytical Methods, and Rational Materials Design, Adv. Sci., 2018, 5(1), 1700322.

63 Z. Li, S. Gadipelli, Y. Yang, G. He, J. Guo, J. Li, Y. Lu, C. A. Howard, D. J. L. Brett, I. P. Parkin, F. Li and Z. Guo, Exceptional supercapacitor performance from optimized 
oxidation of graphene-oxide, Energy Storage Mater., 2019, 17, 12-21.

64 T.-F. Yeh, S.-J. Chen, C.-S. Yeh and H. Teng, Tuning the Electronic Structure of Graphite Oxide through Ammonia Treatment for Photocatalytic Generation of H-2 and O-2 from Water Splitting, J. Phys. Chem. C, 2013, 117(13), 6516-6524.

65 D. R. Dreyer, S. Park, C. W. Bielawski and R. S. Ruoff, The chemistry of graphene oxide, Chem. Soc. Rev., 2010, 39(1), 228-240. 66 Y. Bai, M. Du, J. Chang, J. Sun and L. Gao, Supercapacitors with high capacitance based on reduced graphene oxide/carbon
nanotubes/NiO composite electrodes, J. Mater. Chem. A, 2014, 2(11), 3834-3840.

67 G. Cheng, Y. Yan and R. Chen, From Ni-based nanoprecursors to NiO nanostructures: morphology-controlled synthesis and structure-dependent electrochemical behavior, New J. Chem., 2015, 39(1), 676-682.

68 P. He, J. Sun, S. Tian, S. Yang, S. Ding, G. Ding, X. Xie and M. Jiang, Processable Aqueous Dispersions of Graphene Stabilized by Graphene Quantum Dots, Chem. Mater., 2015, 27(1), 218-226. 